\title{
Gastrointestinal Stromal Tumor: An Uncommon but Serious Cause of Gastrointestinal Bleeding
}

\author{
Firas Alawawdeh ${ }^{\mathrm{a}}$, Amna Al-Tkrit ${ }^{\mathrm{a}}$, Mohammad Aneeb ${ }^{\mathrm{a}}$, \\ Andrew Mekaiel ${ }^{\mathrm{a}, \mathrm{c}}$, Asit Mehta ${ }^{\mathrm{a}, \mathrm{b}}$
}

\begin{abstract}
Gastrointestinal stromal tumors (GISTs) are rare tumors of the GI tract that can sometimes present as a gastrointestinal bleeding source. This report describes a patient presented with upper GI bleeding secondary to a gastric GIST, which was initially detected on endoscopy. The patient underwent surgical resection of the tumor and was started on adjuvant chemotherapy with imatinib. The patient's postoperative course was complicated by an esophageal leak requiring re-exploratory laparotomy and esophageal stent placement. The stent was removed 5 weeks later and the patient remained stable for discharge after 60 days of inpatient care.
\end{abstract}

Keywords: Gastrointestinal stromal tumor; KIT; DOG1; Upper GI bleeding; Imatinib; Tyrosine kinase inhibitor; Immunohistochemistry; Endoscopy

\section{Introduction}

Gastrointestinal stromal tumors (GISTs) are rarely occurring mesenchymal tumors of the digestive tract that originate from interstitial cells of Cajal (ICC). GISTs may be asymptomatic or may present with nonspecific symptoms, such as nausea, vomiting, abdominal pain, or early satiety. However, in some patients, GISTs can be a source of intraperitoneal hemorrhage or can cause bleeding into the GI tract lumen, resulting in melena, hematemesis, or anemia. In this report, we present the case of a patient who developed upper GI bleeding secondary to a high-grade gastric GIST, which was found to be positive for KIT (CD117) and DOG1 on immunohistochemical (IHC) analysis.

Manuscript submitted December 5, 2020, accepted December 15, 2020

Published online December 30, 2020

aDepartment of Internal Medicine, Jamaica Hospital, Queens, NY, USA bepartment of Gastroenterology, Jamaica Hospital, Queens, NY, USA ${ }^{\mathrm{c} C}$ Corresponding Author: Andrew Mekaiel, Department of Internal Medicine, Jamaica Hospital, Queens, 8900 Van Wyck Expy, Richmond Hill, NY 11418, USA. Email: andrewmekaiel@gmail.com

doi: https://doi.org/10.14740/jmc3631

\section{Case Report}

A 43-year-old man with a history of melena and significant anemia presented to the Gastroenterology Department of our hospital for outpatient esophagogastroduodenoscopy (EGD).

The patient noticed melanotic stools about 2 weeks before presentation, and around the same time, he started experiencing dizziness, generalized weakness, and intermittent epigastric pain. He denied any weight loss, loss of appetite, or night sweats. The patient was a former smoker and had quit smoking cigars almost 2 years ago. He was a social drinker and denied illicit drug use. Family history was significant for colon cancer in his maternal uncle.

EGD performed by the gastroenterologist showed a large submucosal mass with central ulceration located in the proximal gastric body. A biopsy of the gastric tissue was obtained. The patient developed acute GI bleeding after the biopsy was taken. Endoscopic hemostasis was successfully achieved with epinephrine injections; however, the patient had to be transfused with multiple units of packed red blood cells due to significant blood loss and anemia. He was then admitted to the medical intensive care unit (MICU) for close monitoring.

Computed tomography (CT) scan of the abdomen and pelvis with intravenous (IV) and per os (PO) contrast revealed a $19 \times 16 \times 13 \mathrm{~cm}$ mass located in the left upper quadrant. The mass appeared to originate from the stomach's greater curvature and was predominantly necrotic/cystic in nature, with enhancing thick internal septations. The gastric biopsy results showed moderate chronic active gastritis with lymphoid aggregation, and the biopsy specimen was found to be Helicobacter pylori (H. pylori) positive. The patient was started on triple therapy and was taken to surgery for abdominal mass resection, partial gastrectomy, distal pancreatectomy, and splenectomy. The postoperative course was complicated by an esophageal leak requiring re-exploratory laparotomy and esophageal stent placement. The stent was removed 5 weeks later as the esophagogram showed no leak at that time.

The histopathology report of the left upper quadrant mass was consistent with a GIST $18 \times 15 \times 12 \mathrm{~cm}$ in diameter (Fig. 1). The positive IHC staining for KIT (CD117) and DOG1 (ANO1) confirmed the diagnosis (Figs. 2, 3). The histology of gastric mucosa revealed chronic gastric inflammation with lymphoid aggregation; whereas, that of the spleen demonstrated focal disruption and hemorrhage without any evidence of tumor involvement. The omentum showed focal congestion, 


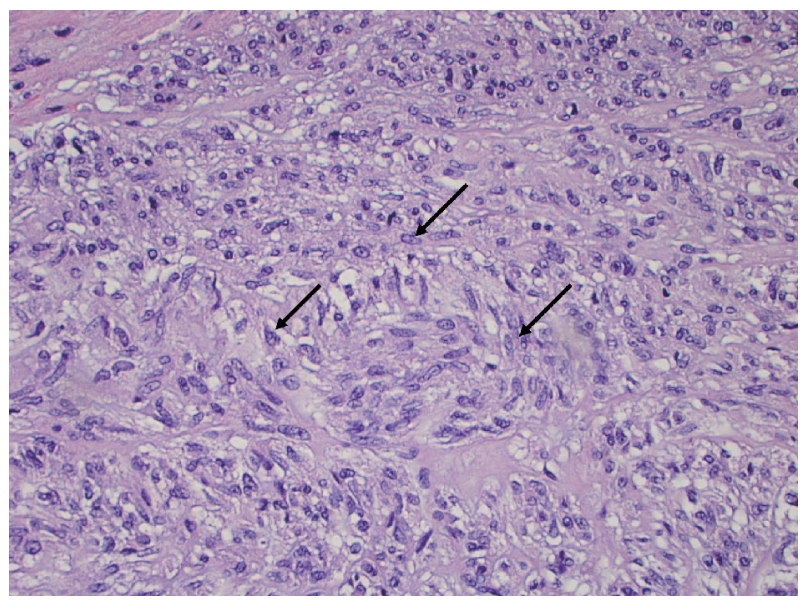

Figure 1. Spindle cells (arrow) proliferation in gastric wall (hematoxylin and eosin stain, $400 \times$ magnification).

hemorrhage, and fibrosis, but no histologic evidence of tumor involvement was found in the omentum as well.

An oncology consultation was obtained. The initial clinical impression was that the patient had a high-grade GIST with a high mitotic index. The patient underwent staging and was found to have no regional lymph node metastasis. CT chest was also negative for metastasis. Adjuvant chemotherapy with imatinib $400 \mathrm{mg}$ daily was initiated. The patient had a prolonged hospital course of around 60 days, during which he received postoperative care, nutritional support, and physical therapy. He was subsequently discharged from the hospital in a stable condition and was advised to follow up with his primary care physician, as well as the oncology and surgery departments.

\section{Discussion}

GISTs are rare neoplasms of the GI tract and account for up to $1-2 \%$ of all GI malignancies [1]. However, they are the most common mesenchymal tumors of the alimentary canal, making

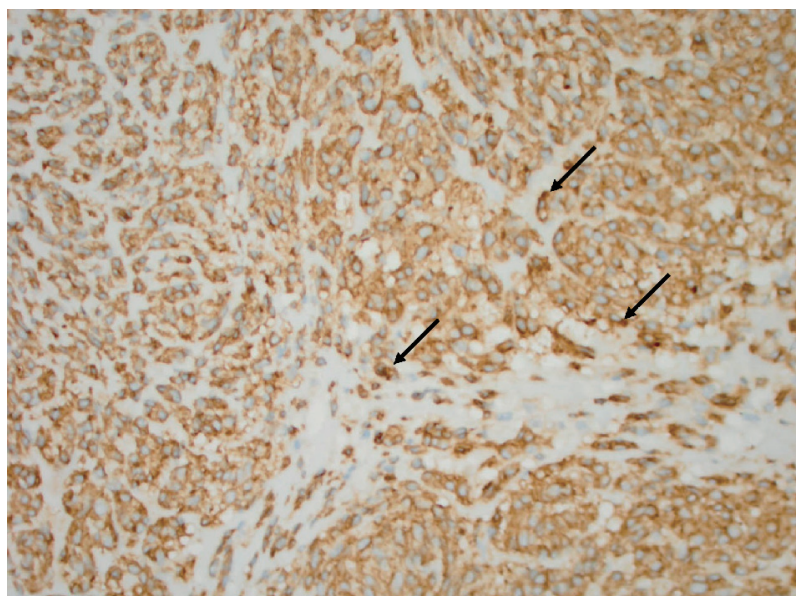

Figure 2. Tumor cells (arrow) with positive cytoplasmic CD117 antibody stain (immunohistochemistry stain, $400 \times$ magnification).

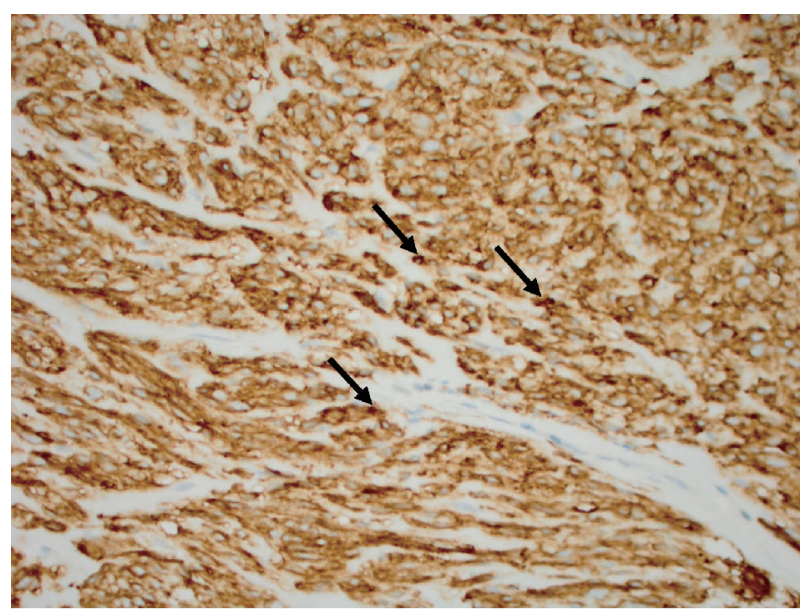

Figure 3. Tumor cells (arrow) with positive cytoplasm DOG1 antibody stain (immunohistochemistry stain, $400 \times$ magnification).

up almost $80 \%$ of all GI mesenchymal tumors. The estimated incidence of GISTs is between 10 and 15 cases per million. The incidence has been increasing; but, this may be secondary to an improved diagnostic ability rather than an actual increase in incidence [2]. GIST is a disease of older adults, and the median age at diagnosis is 65 years, with only less than $10 \%$ of patients diagnosed before the age of 40 [3].

GISTs were initially believed to have originated from smooth muscle cells and were classified as smooth muscle neoplasms [4]. Mazur and Clark were the first to describe "gastric stromal tumors" as a separate entity distinct from the gastric smooth muscle tumors in 1983 [5]. In the year 1998, it was reported that GISTs originated from the ICC [6]. GISTs can occur throughout the entire GI tract, with the stomach (56\%) being the most common location, followed by the small bowel $(32 \%)$, colorectum $(6 \%)$, and esophagus $(<1 \%)$ [2]. Around $5 \%$ of GISTs may be extragastrointestinal. These are known as extragastrointestinal GISTs or EGISTs and usually affect the omentum, mesentery, and retroperitoneum [7].

KIT is a glycoprotein that belongs to the type III transmembrane receptor tyrosine kinase (RTK) family, and mutations in the KIT gene are considered the most common pathogenetic changes involved in the development of GISTs. KIT mutations cause constitutive activation of the tyrosine kinase, resulting in the uncontrolled proliferation of Cajal's interstitial cells and the subsequent development of GISTs. KIT-activating mutations are found in approximately $70-80 \%$ of GISTs. About $5-10 \%$ of GISTs lack KIT gene mutations but have been reported to have mutations in PDGFRA, a close homolog of KIT. The oncogenic mechanisms appear to be alternative, and the mutations in GIST and PDGFRA are mutually exclusive. Approximately $9-15 \%$ of GISTs lack detectable mutations in either KIT or PDGFRA, and are termed as "wild-type" GISTs. There is no clinical difference between wild-type GISTs and KIT- or PDGFRA-mutant GISTs. Wild-type GISTs may harbor a few other genetic mutations, such as BRAF, NRAS, HRAS, and PIK3CA gene mutations. Most GISTs occur sporadically, and familial GISTs with germline mutations of KIT or $P D G$ $F R A$ are extremely rare $(<0.1 \%)$. GISTs may also develop in 
association with specific clinical syndromes, such as neurofibromatosis type 1 (NF1), Carney-Stratakis syndrome, and Carney triad $[4,8]$.

GISTs can range from a few millimeters to over $30 \mathrm{~cm}$, with $5-8 \mathrm{~cm}$ being the median size. Around $20 \%$ of patients with GISTs are asymptomatic, and the tumors are diagnosed incidentally. Seventy percent of patients may present with symptoms; however, the symptoms are not disease-specific and vary according to the tumor's size and location. These symptoms may include abdominal pain, nausea, vomiting, early satiety, or weight loss. GI bleeding is the most common complication associated with GISTs and may be seen in 30$40 \%$ of patients. Mucosal ulceration or tumor rupture may result in intraperitoneal hemorrhage presenting as a life-threatening surgical emergency. Bleeding into the GI tract lumen, on the other hand, may lead to melena, hematemesis, or anemia and usually has a more chronic presentation [9]. Intraluminal hemorrhage may be caused by mucosal and submucosal destruction secondary to tumor growth, tumor necrosis, tumor invasion of nutrient vessels resulting in vascular rupture, and the combined action of GI peristalsis, digestive juices, and fecal transmission. It has been reported that GISTs are more likely to cause bleeding in the small intestine than in the stomach. This may be related to the larger size of the stomach as compared to the small intestine. Distant metastasis occurs most commonly to the liver, peritoneum, and greater omentum. Lymph node metastasis is uncommon [10].

The diagnostic evaluation of GISTs is based on imaging modalities, and most tumors are detected by endoscopy. On endoscopy, a GIST typically appears as a well-circumscribed spherical or hemispheric mass, originating primarily from underneath the mucosa and bulging into the lumen. Endoscopic ultrasonography (EUS) is highly helpful, particularly when the GIST is relatively small in size and lacks a non-significant outward expansion. CT scan of the abdomen/pelvis is the best imaging method for diagnosing and staging GISTs. On CT scan, small GISTs usually appear as well-defined soft tissue masses that show moderate contrast enhancement. Larger tumors may have central necrosis and cavitation along with heterogeneous enhancement. Magnetic resonance imaging (MRI) provides similar information as CT but may be more accurate in identifying anorectal GISTs and metastatic hepatic GISTs. On MRI, GISTs typically show low to intermediate signal intensity on T1-weighted images and are hyperintense on T2-weighted images [11-13].

The pathological diagnosis of GISTs is established based on morphology and IHC findings. There are three main morphological types of GISTs. These include spindle cell type, epithelioid type, and mixed type. The spindle cell type GISTs account for $70 \%$ of all GISTs. They are composed of spindleshaped cells with fibrillary eosinophilic cytoplasm, arranged in a fascicular pattern with minimal stroma. The epithelioid type GISTs are made up of cells with abundant, pale eosinophilic or clear cytoplasm. These cells are arranged in sheets, nests, or cords. Twenty percent of GISTs are of the epithelioid type. The mixed type GISTs consist of spindle cells and epithelioid cells and account for $10 \%$ of all GISTs [4]. GIST histology may show a variable inflammatory infiltrate, hemorrhage, and necrosis, irrespective of the morphological type. Tumors may exhibit a complete absence of mitotic activity or they may be highly active. Mitotic index is used for the grading of a GIST and is an important prognostic indicator [8]. Immunohistochemically, around $95 \%$ of GISTs are strongly positive for KIT (CD117), and discovered on GIST-1 (DOG1), also known as anoctamin-1 (ANO1). Approximately $70-80 \%$ of GISTs co-express CD34; however, it is less sensitive and specific. GISTs may also be positive for smooth muscle actin in $30-40 \%$ of the cases and may rarely be positive for $\mathrm{S} 100$ protein (Schwann cell marker), desmin (smooth muscle marker), and keratin [4, 13].

Surgical resection is the only potentially curative treatment of GISTs. It is recommended for GISTs $\geq 2 \mathrm{~cm}$ in diameter and GISTs with high-risk malignant features on EUS, such as size $\geq 2 \mathrm{~cm}$, irregular borders, and the presence of ulceration, echogenic foci, anechoic spaces, or heterogeneous echogenicity. Surgery is also the treatment of choice for localized, symptomatic GISTs, with emergent surgery required for bleeding gastric GISTs. The surgical approach depends on the size, location, and number of gastric GISTs. The various surgical options include wedge partial resection of the stomach, Billroth I partial gastrectomy, Billroth II partial gastrectomy, or total gastrectomy with Roux-en-Y reconstruction. GISTs rarely metastasize to the lymph nodes, and so, regional lymph node dissection is not usually needed. Endoscopic resection methods, such as endoscopic enucleation, endoscopic band ligation, and endoscopic submucosal dissection are primarily used for GISTs of the upper GI tract in patients who do not have metastatic or recurrent disease $[8,10]$.

However, the likelihood of developing a recurrent disease or metastasis even after complete resection of GIST is around 40-50\% [14]. High-risk factors associated with recurrence include tumor size $>5 \mathrm{~cm}$, mitotic count $>5$ per 50 highpower fields (HPFs), location of the tumor (small bowel or rectal GISTs have a worse prognosis than gastric GISTs), and tumor rupture. Also, GI bleeding is associated with a higher risk of recurrence $[14,15]$. Patients at increased risk for recurrence are treated with adjuvant chemotherapy with imatinib, a tyrosine kinase inhibitor, which has been found to reduce recurrence risk. Imatinib is also used in the treatment of unresectable, recurrent, or metastatic disease [16]. For imatinibresistant or intolerant patients with advanced disease, sunitinib and regorafenib have shown efficacy as second- and third-line agents, respectively [17].

GISTs are uncommon yet important tumors of the digestive tract. Small GISTs may be asymptomatic; however, larger tumors may present serious clinical manifestations, such as GI bleeding. Our patient presented with a history of upper GI bleeding was found to have a large, submucosal, gastric GIST. The tumor was surgically resected and was found to be positive for KIT (CD117) and DOG1 on immunohistochemical analysis. The patient was started on adjuvant chemotherapy with imatinib. This case highlights the fact that even if rare, GISTs must be included in the differential diagnosis of GI bleeding so that an early diagnosis can be established and appropriate treatment can be initiated promptly. Due to a high risk of recurrence of GISTs, long-term, intense follow-up is required for all patients, particularly for high-risk patients. An annual postoperative follow-up examination and an abdominopelvic CT scan every 3 to 6 months for a period of 3 to 5 years is the 
recommended surveillance protocol [18]. Less frequent observation may be acceptable for GISTs $<2 \mathrm{~cm}$ in diameter $[9,12]$.

\section{Conclusions}

GISTs are rare and may often be asymptomatic or present with nonspecific symptoms; however, they can sometimes be a source of potentially life-threatening GI bleeding and should, therefore, be considered as an important differential diagnosis of GI bleeding. They are typically diagnosed using imaging studies and have distinct histopathologic and immunohistochemical features. Surgical resection remains the treatment of choice for localized, symptomatic GISTs, including gastric GISTs presenting with GI bleeding. Imatinib is often used as adjuvant therapy to reduce the risk of recurrence. Long-term follow-up with clinical examination and abdominopelvic CT imaging is recommended.

\section{Acknowledgments}

None to declare.

\section{Financial Disclosure}

All authors declare that no financial support was received from any organization for the submitted work.

\section{Conflict of Interest}

None to declare.

\section{Informed Consent}

No PHI shared in this publication as per HIPAA guidelines. The consent was obtained in this study.

\section{Author Contributions}

Firas Alawawdeh MD as the first author: manuscript preparation and writing, literature searches and review. Amna Al-Tkrit MD: manuscript preparation and writing, case study, literature searches and review. Mohammad Aneeb MD: manuscript preparation, case summary, literature searches and review. Andrew Mekaiel MD: manuscript preparation and submission, case summary, literature searches and review. Asit Mehta MD: manuscript final review, literature searches and review.

\section{Data Availability}

The authors declare that data supporting the findings of this study are available within the article.

\section{References}

1. Parab TM, DeRogatis MJ, Boaz AM, Grasso SA, Issack PS, Duarte DA, Urayeneza O, et al. Gastrointestinal stromal tumors: a comprehensive review. J Gastrointest Oncol. 2019;10(1):144-154.

2. Sanchez-Hidalgo JM, Duran-Martinez M, Molero-Payan R, Rufian-Pena S, Arjona-Sanchez A, Casado-Adam A, Cosano-Alvarez A, et al. Gastrointestinal stromal tumors: A multidisciplinary challenge. World J Gastroenterol. 2018;24(18):1925-1941.

3. NS IJ, Drabbe C, den Hollander D, Mohammadi M, van Boven H, Desar IME, Gelderblom H, et al. Gastrointestinal stromal tumours (GIST) in young adult (18-40 Years) patients: a report from the Dutch GIST registry. Cancers (Basel). 2020;12(3):730.

4. Zhao X, Yue C. Gastrointestinal stromal tumor. J Gastrointest Oncol. 2012;3(3):189-208.

5. Mazur MT, Clark HB. Gastric stromal tumors. Reappraisal of histogenesis. Am J Surg Pathol. 1983;7(6):507-519.

6. Kindblom LG, Remotti HE, Aldenborg F, Meis-Kindblom JM. Gastrointestinal pacemaker cell tumor (GIPACT): gastrointestinal stromal tumors show phenotypic characteristics of the interstitial cells of Cajal. Am J Pathol. 1998;152(5):1259-1269.

7. Nguyen Thanh T, Nguyen TTN, Le TB, Le DD, Nguyen VM, Le DK. Extragastrointestinal stromal tumor presenting as an exophytic prostatic mass. Radiol Case Rep. 2020;15(8):1142-1148.

8. Ahmed M. Recent advances in the management of gastrointestinal stromal tumor. World J Clin Cases. 2020;8(15):3142-3155.

9. Rammohan A, Sathyanesan J, Rajendran K, Pitchaimuthu A, Perumal SK, Srinivasan U, Ramasamy R, et al. A gist of gastrointestinal stromal tumors: A review. World J Gastrointest Oncol. 2013;5(6):102-112.

10. Liu Q, Kong F, Zhou J, Dong M, Dong Q. Management of hemorrhage in gastrointestinal stromal tumors: a review. Cancer Manag Res. 2018;10:735-743.

11. Mokhtare M, Taghvaei T, Tirgar Fakheri H. Acute bleeding in duodenal gastrointestinal stromal tumor. Middle East J Dig Dis. 2013;5(1):47-51.

12. Marcella C, Shi RH, Sarwar S. Clinical overview of GIST and its latest management by endoscopic resection in upper GI: a literature review. Gastroenterol Res Pract. 2018;2018:6864256.

13. Rare Tumors GI Group, Farhat F, Farsi AA, Mohieldin A, Bahrani BA, Sbaity E, Jaffar H, et al. Comprehensive review into the challenges of gastrointestinal tumors in the Gulf and Levant countries. World J Clin Cases. 2020;8(3):487-503.

14. Liu Q, Li Y, Dong M, Kong F, Dong Q. Gastrointestinal bleeding is an independent risk factor for poor prognosis in GIST patients. Biomed Res Int. 2017;2017:7152406.

15. Huang Y, Zhao R, Cui Y, Wang Y, Xia L, Chen Y, Zhou $\mathrm{Y}$, et al. Effect of gastrointestinal bleeding on gastrointestinal stromal tumor patients: a retrospective cohort study. Med Sci Monit. 2018;24:363-369. 
16. Laurent M, Brahmi M, Dufresne A, Meeus P, Karanian M, Ray-Coquard I, Blay JY. Adjuvant therapy with imatinib in gastrointestinal stromal tumors (GISTs)-review and perspectives. Transl Gastroenterol Hepatol. 2019;4:24.

17. Balachandran VP, DeMatteo RP. Gastrointestinal stromal tumors: who should get imatinib and for how long? Adv
Surg. 2014;48:165-183.

18. Demetri GD, von Mehren M, Antonescu CR, DeMatteo RP, Ganjoo KN, Maki RG, Pisters PW, et al. NCCN Task Force report: update on the management of patients with gastrointestinal stromal tumors. J Natl Compr Canc Netw. 2010;8(Suppl 2):S1-41; quiz S42-44. 